\title{
Comparison of loss of response between anti-tumor necrosis factor alone and combined use with immunomodulators in patients with inflammatory bowel disease
}

\author{
Seung Wook Hong ${ }^{1,{ }^{*}}$, Jaewoo Park ${ }^{2,}$, Hyuk Yoon $^{2}$, Hye Ran Yang ${ }^{3}$, Cheol Min Shin ${ }^{2}$, Young Soo Park ${ }^{2}$, \\ Nayoung Kim², Dong Ho Lee ${ }^{2}$, and Joo Sung Kim
}

${ }^{1}$ Department of Internal Medicine and Liver Research Institute, Seoul National University College of Medicine, Seoul; Departments of ${ }^{2}$ Internal Medicine and ${ }^{3}$ Pediatrics, Seoul National University Bundang Hospital, Seongnam, Korea

Received: August 22, 2019 Revised : January 13, 2020 Accepted: January 17, 2020

\section{Correspondence to} Hyuk Yoon, M.D.

Division of Gastroenterology, Department of Internal Medicine, Seoul National University Bundang Hospital, 82 Gumiro173 Beon-gil, Bundang-gu, Seongnam 13620, Korea Tel: +82-31-787-7009 Fax: $+82-31-787-4051$ E-mail: bodnsoul@hanmail.net https://orcid.org/0000-00022657-0349

*These authors contributed equally to this work.
Background/Aims: Combination therapy with immunomodulators (IMMs) was proposed as a strategy to prevent the development of loss of response (LOR) to anti-tumor necrosis factor (TNF) for patients with inflammatory bowel disease (IBD). However, the effect is unclear in patients already exposed to IMMs. The aim of this study was to evaluate whether combination therapy with IMMs is superior to monotherapy for prevention of LOR to anti-TNF.

Methods: This was a retrospective study of patients in Seoul National University Bundang Hospital with IBD between January 2009 and October 2018. LOR was defined as clinical deterioration after maintenance of anti-TNF for at least 6 months. We investigated the difference in incidence of LOR to anti-TNF between the monotherapy and combination groups. We additionally assessed factors affecting LOR development to anti-TNF.

Results: A total of 116 patients with IBD were included in this study (monotherapy 61 patients; combination 55 patients). Overall, LOR to anti-TNF occurred in 31 patients during the follow-up period. The combination of an anti-TNF agent and IMM showed no significant difference in the incidence of LOR compared to anti-TNF agent monotherapy (hazard ratio [HR], 1.64; 95\% confidence interval [CI], 0.786 to $3.148 ; p=0.182$ ). Female sex was significantly associated with the development of LOR to anti-TNF (HR, 3.032; 95\% CI, 1.467 to $6.268 ; p=0.003$ ).

Conclusions: Anti-TNF and IMM combination therapy did not prove efficacious in preventing the development of LOR in IBD patients. Female sex was associated with the development of LOR to anti-TNF; further studies are required to confirm these results.

Keywords: Colitis, ulcerative; Crohn disease; Infliximab; Immunosuppressive agents; Inflammatory bowel diseases

\section{INTRODUCTION}

Inflammatory bowel disease (IBD) is a chronic inflam- matory disease, primarily involving the intestine, which is caused by the interaction of various factors [1]. Since their introduction, biologic agents have become the 
mainstream treatment of IBD. However, as the use of biologics, especially anti-tumor necrosis factor (TNF) agents, increases in IBD patients, loss of response (LOR) to the agents has emerged as a new obstacle for clinicians [2]. There is no consensus regarding the definition of LOR to anti-TNF agents, but most cases are described as patients who responded during the induction period but had LOR during the maintenance period $[2,3]$.

The formation of antibodies against anti-TNF agents, immunogenicity, is the key mechanism in the development of LOR $[2,3]$. These antibodies interfere with the action of anti-TNF agents and are associated with lower serum drug levels. Hence, combination therapy with immunomodulators (IMMs) has been proposed as a strategy to prevent the development LOR to anti-TNF agents in IBD patients $[2,3]$. However, it is not clear whether combination therapy is helpful in reducing the incidence of LOR in all IBD patients. In the Study of Biologic and Immunomodulator Naive Patients in Crohn Disease (SONIC) trial, infliximab combined with azathioprine showed a clinical benefit compared to that of monotherapy for patients with Crohn's disease (CD) at 12 months, but the study population was limited to IMM naïve patients [4]. Similarly, for patients with ulcerative colitis (UC), treatment with infliximab plus an IMM showed superior outcomes compared to that of monotherapy, however, most of the patients enrolled had not been exposed to IMMs [5]. Moreover, a meta-analysis of CD patients for whom IMM treatment failed, combination therapy was not found to be more effective than monotherapy in response rates at 6 months [6]. These findings suggest that exposure to IMMs can be a crucial factor in determining the responsiveness of combination therapy. On the other hand, the long-term effect of the combination therapy with an anti-TNF agent and IMM is still controversial. In a study by Billiet et al. [7], concomitant with an IMM was not favorable with regards to long-term outcomes for patients with CD. In addition, a recent long-term retrospective cohort study revealed that there is no difference in the development of LOR to anti-TNF agents between monotherapy and combination IMM therapy [8].

Given the conflicting results of previous research, the aim of this study was to confirm whether combination therapy with an IMM is superior to monotherapy for the prevention of LOR to anti-TNF agents. In addition, factors associated with the development of LOR to an-
ti-TNF agents were investigated.

\section{METHODS}

\section{Study design and population}

This was a retrospective cohort study of patients diagnosed with CD or UC in Seoul National University Bundang Hospital (SNUBH) between January 2009 and October 2018. We included patients who started and continued anti-TNF agents for at least 6 months. There was no restriction on the type of anti-TNF agent. We retrospectively collected demographic data (sex, age, etc.) and clinical information including drug history and laboratory results from patient medical records. This study was approved by the Institutional Review Board at SNUBH, and the requirement for informed consent was waived (IRB approved date: 2019.03.23, No. of IRB: L2019-248).

\section{Definition and outcomes}

We defined LOR to anti-TNF as a patient who showed clinical deterioration after maintenance of anti-TNF agents for at least 6 months. The clinical deteriorations included increased inflammatory markers as well as aggravation of gastrointestinal symptoms described by the patient. Exposure to IMMs was defined as taking them before commencement of taking the anti-TNF agent. We stratified the included patients according to whether an anti-TNF agent and IMM were used in combination or not. We assigned the patients who received IMM combination treatment with the commencement of an anti-TNF agent and continued IMM use for at least 6 months to the combination group, and those who received an anti-TNF agent alone without an IMM to the monotherapy group.

We investigated the difference in the incidence of LOR to anti-TNF between the monotherapy group and combination group. Additionally, we assessed the factors affecting the development of LOR to anti-TNF.

\section{Statistical analysis}

We compared the characteristics between the monotherapy group and combination group using the Student $t$ test for continuous variables, and the Pearson chi-square test or the Fisher exact test for categorical 
variables. A p value of less than 0.05 was considered to be statistically significant. The Kaplan-Meier method was used to analyze the incidence of LOR to anti-TNF and the difference in treatment strategies was compared by the log-rank test. Subgroup analysis divided by the type of IBD was performed. The propensity matching analysis was additionally carried out by using the one-to-one nearest-neighbor matching techniques with a 0.01 caliper level. The risk factors for LOR to anti-TNF were analyzed using the Cox proportional hazard model. All variables associated with LOR to anti-TNF in univariate Cox models and only variables with a $p<0.25$ were included in multivariable models. The results are presented with hazard ratios (HRs) with 95\% confidence intervals (CIs). All statistical analyses were performed using the R software version 3.5.3. (The R foundation, Vienna, Austria)

\section{RESULTS}

\section{Baseline characteristics in monotherapy and combi- nation group}

A total of 116 patients with IBD were included in this study; 61 patients and 55 patients with IBD were assigned to the monotherapy group and combination group, respectively. The baseline characteristics in the monotherapy and combination groups are summarized in Table 1. The average age of patients with IBD was 37.1 \pm 15.6 years in the monotherapy group and $30.6 \pm 13.4$ years in the combination group $(p=0.018)$. The age at diagnosis of the enrolled patients was similar in both groups (monotherapy: $27.2 \pm 14.4$ years vs. combination: $23.7 \pm 12.5$ years, $p=0.177$ ). There was no significant difference in the body weight between the two groups at initiation of the anti-TNF agents (monotherapy: 44.1 \pm $24.7 \mathrm{~kg}$ vs. combination: $51.9 \pm 25.9 \mathrm{~kg}, p=0.100$ ). The follow-up duration of the monotherapy group was significantly longer than that of the combination group (total: $158.99 \pm 89.6$ weeks, monotherapy: $175.5 \pm 100.8$ weeks vs. combination: $140.7 \pm 71.9$ weeks, $p=0.033$ ). The disease duration was also significantly longer in the monotherapy group compared with the combination group (monotherapy: $399.7 \pm 310.7$ weeks vs. combination: 272.8 \pm 214.4 weeks, $p=0.013$ ) .

The proportion of patients with different IBD types was similar in both groups. There were $42 \mathrm{CD}$ patients
(68.9\%) and 19 UC patients (31.1\%) in the monotherapy group and $38 \mathrm{CD}$ patients (69.1\%) and $17 \mathrm{UC}$ patients (30.9\%) in the combination group. The enrolled patients were prescribed anti-TNF agents including infliximab, adalimumab, and golimumab. Infliximab was the most frequently used in each group ( $85.2 \%$ vs. $78.2 \%$ ) and there was no significant difference in the proportion of anti-TNF types prescribed between the two groups $(p=$ 0.577). The mean dosage of IMMs in the combination group was as follows (azathioprine: $58.55 \mathrm{mg}$, 6-mercaptopurine: $25.0 \mathrm{mg}$, and methotrexate: $14.53 \mathrm{mg}$ ). The concomitant medications used in both groups were 5-aminosalicylic acid (5-ASA) and sulfasalazine. The proportion of concomitant medications was not significantly different between the two groups (5-ASA: 63.9\% vs. $50.9 \%$; sulfasalazine: $3.3 \%$ vs. $5.5 \%, p=0.356$ ). IMMs were not maintained for the long-term in the monotherapy group due to side effects $(n=20)$ and refractoriness of IMMs $(n=9)$. There were more IMM naïve patients in the monotherapy group than in the combination group (52.5\% vs. $16.4 \%, p<0.001$ ). Steroid exposure and history of abdominal surgery were similar in both groups $(p=$ 0.486 and $p=0.121$, respectively). During the follow-up duration, a total of 35 patients stopped anti-TNF agent treatment due to the occurrence of LOR or adverse events. There was no significant difference between the two groups with regards to the reason for discontinuation $(p=0.422)$.

\section{IBD classification and severity index according to treatment strategy}

The included patients were further subdivided by IBD type (CD or UC). The IBD classification and severity index are presented in Table 2. IBD classification was based on the Montreal classification of IBD and the severity was assessed using a scale appropriate for the type of IBD and age [9].

Of the 80 patients with $\mathrm{CD}, 42$ patients were assigned to the monotherapy group and 38 patients to the combination group. In both groups, the proportions in terms of age at onset, disease location, disease behavior, and presence of perianal disease were similar. There were no significant differences in severity index at onset for adults $(344.8 \pm 94.0$ vs. $295.8 \pm 90.0, p=0.072)$ or pediatrics $(29.2 \pm 12.3$ vs. $32.4 \pm 13.8, p=0.602)$ between each group. Of the $36 \mathrm{UC}$ patients, 19 were assigned to the 
Table 1. Baseline characteristics of patients in anti-TNF combination and monotherapy groups

\begin{tabular}{|c|c|c|c|}
\hline Characteristic & Monotherapy $(n=61)$ & Combination $(\mathrm{n}=55)$ & $p$ value \\
\hline Age, yr & $37.1 \pm 15.6$ & $30.6 \pm 13.4$ & $0.018^{\mathrm{a}}$ \\
\hline Age at diagnosis, yr & $27.2 \pm 14.4$ & $23.7 \pm 12.5$ & 0.177 \\
\hline Sex, male & $36(59.0)$ & $38(69.1)$ & 0.253 \\
\hline Body weight at time of initiation, $\mathrm{kg}$ & $44.1 \pm 24 \cdot 7$ & $51.9 \pm 25.9$ & 0.100 \\
\hline IBD, type & & & NS \\
\hline Crohn's disease & $42(68.9)$ & $38(69.1)$ & \\
\hline Ulcerative colitis & $19(31.1)$ & $17(30.9)$ & \\
\hline Follow-up duration, wk & $175 \cdot 5 \pm 100.8$ & $140.7 \pm 71.9$ & $0.033^{\mathrm{a}}$ \\
\hline Disease duration, wk & $399.7 \pm 310.7$ & $272.8 \pm 214.4$ & $0.013^{\mathrm{a}}$ \\
\hline Type of anti-TNF & & & 0.577 \\
\hline Infliximab & $52(85.2)$ & $43(78.2)$ & \\
\hline Adalimumab & $8(13.1)$ & $10(18.2)$ & \\
\hline Golimumab & $1(1.6)$ & $2(3.6)$ & \\
\hline \multicolumn{4}{|l|}{ Immunomodulator, type } \\
\hline Azathioprine & NA & $42(76.4)$ & \\
\hline 6-Mercaptopurine & NA & $11(20.0)$ & \\
\hline Methotrexate & NA & $2(3.6)$ & \\
\hline Concomitant medication & & & 0.356 \\
\hline None & $20(32.8)$ & $24(43.6)$ & \\
\hline 5 -ASA & $39(63.9)$ & $28(50.9)$ & \\
\hline Sulfasalazine & $2(3 \cdot 3)$ & $3(5 \cdot 5)$ & \\
\hline IMM naïve & $32(52 \cdot 5)$ & $9(16.4)$ & $<0.001^{\mathrm{a}}$ \\
\hline Steroid exposure & $56(91.8)$ & $53(96.4)$ & 0.486 \\
\hline History of abdominal surgery & $2(3 \cdot 3)$ & $7(12.7)$ & 0.121 \\
\hline Anti-TNF discontinuation & $16(26.2)$ & $19(34 \cdot 5)$ & 0.422 \\
\hline Loss of response & $15(24.6)$ & $16(29.1)$ & \\
\hline Adverse events & $1(1.6)$ & $3(5 \cdot 5)$ & \\
\hline
\end{tabular}

Values are presented as mean $\pm \mathrm{SD}$ or number $(\%)$.

TNF, tumor necrosis factor; IBD, inflammatory bowel disease; NS, not significant; NA, not available; 5-ASA,5-aminosalicylic acid; IMM, immunomodulator.

$\mathrm{a} p<0.05$.

monotherapy and 17 to the combination group. As with the CD findings, no significant differences were founded regarding the disease extent and severity index at onset between the monotherapy and combination groups.

\section{Incidence rate of LOR to anti-TNF stratified by the treatment strategy}

A total of 31 patients with IBD had a LOR to anti-TNT during the follow-up period. Of these, 15 patients were in the monotherapy group (35.7\%) and 16 were in the combination group (42.1\%). There was no significant difference in the incidence of LOR to anti-TNF according to the treatment strategy $(p=0.182$ ) (Fig. 1). Further analysis was performed by dividing the groups in accordance with exposure to IMMs. In both the IMM exposure and the IMM naïve group, there was no difference in the rate of LOR development prevention between the combination therapy and monotherapy groups $(p=0.176$ and $p=0.926$, respectively) (Fig. 2). 
Table 2. IBD classification and severity index according to treatment strategy

\begin{tabular}{|c|c|c|c|}
\hline Classification & Monotherapy & Combination & $p$ value \\
\hline Crohn's disease & 42 & 38 & \\
\hline Age at onset & & & 0.857 \\
\hline A1: below 16 years & $14(33.3)$ & $12(31.6)$ & \\
\hline A2: between 17 and 40 years & $26(61.9)$ & $25(68.8)$ & \\
\hline A3: above 40 years & $2(4.8)$ & $1(2.6)$ & \\
\hline Disease location & & & 0.353 \\
\hline Lı: ileal & $7(16.7)$ & $10(26.3)$ & \\
\hline L2: colonic & $10(23.8)$ & $5(13.2)$ & \\
\hline L3: ileocolonic & $25(59 \cdot 5)$ & $23(60.5)$ & \\
\hline L4: isolated upper disease & 0 & 0 & \\
\hline Disease behavior & & & 0.588 \\
\hline $\mathrm{B} 1$ : non-stricturing, non-penetrating & $30(71.4)$ & $23(60.5)$ & \\
\hline B2: stricturing & $4(9 \cdot 5)$ & $5(13.2)$ & \\
\hline B3: penetrating & $8(19.0)$ & $10(26.3)$ & \\
\hline Presence of perianal disease & $17(40.5)$ & $14(36.8)$ & 0.918 \\
\hline \multicolumn{4}{|l|}{ Severity index at onset } \\
\hline CDAI & $344.8 \pm 94.0$ & $295.8 \pm 90.0$ & 0.072 \\
\hline Pediatric CDAI & $29.2 \pm 12.3$ & $32.4 \pm 13.8$ & 0.602 \\
\hline Ulcerative colitis (UC) & 19 & 17 & \\
\hline Disease extent & & & $0.75^{8}$ \\
\hline E1: proctitis & $5(26.3)$ & $3(17.6)$ & \\
\hline E2: left-sided UC & $5(26.3)$ & $4(23.5)$ & \\
\hline E3: extensive UC & $9(47 \cdot 4)$ & $10(58.8)$ & \\
\hline \multicolumn{4}{|l|}{ Severity index at onset } \\
\hline Mayo score & $10.5(9.0-11.0)$ & $9.0(8.00-9.5)$ & 0.198 \\
\hline
\end{tabular}

Values are presented as number (\%), mean $\pm \mathrm{SD}$, or median (interquartile range).

IBD, inflammatory bowel disease; CDAI, Crohn's Disease Activity Index.

\section{Risk factors of LOR to anti-TNF}

To determine the risk factors for the development of LOR to anti-TNF agents, all variables associated with LOR to anti-TNF were included in both univariate and multivariable analysis. The risk factors with a $p<0.25$ in the univariate analysis were included in the multivariable analysis. The results of the analysis are presented in Table 3. In multivariable analysis, female sex was significantly associated with the development of LOR to anti-TNF (HR, 3.032; 95\% CI, 1.467 to $6.268, p=0.003$ ).

\section{DISCUSSION}

In this study, we investigated whether combination therapy with an anti-TNF agent and IMM was more effective than treatment with an anti-TNF agent alone for the prevention of development of LOR to anti-TNF agents for patients with IBD. The results of this study showed that combination therapy with an anti-TNF agent and IMM was not significantly superior to monotherapy with regards to the incidence of LOR to anti-TNF agents. In multivariable analysis, only female sex was significantly associated with the development of LOR to anti-TNF agents for patients with IBD. Although this retrospective 
Probability of LOR to anti-TNF (mono vs. combination therapy)

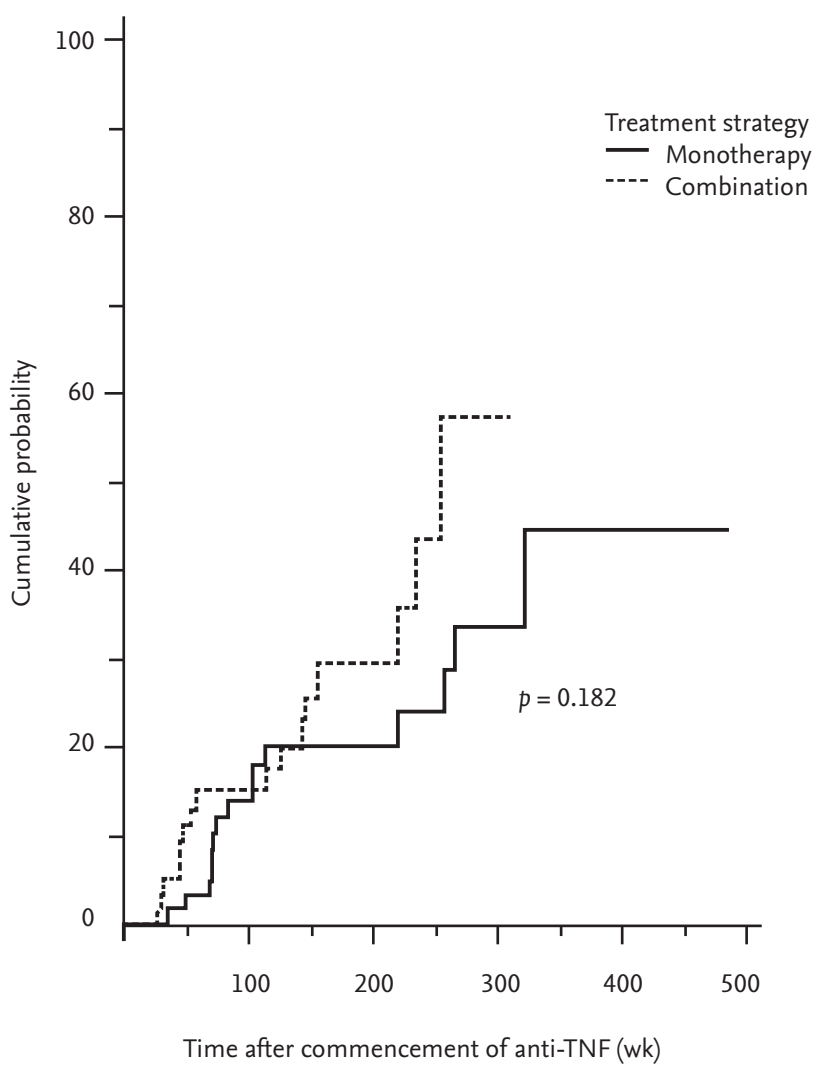

Figure 1. Kaplan-Meier curve shows the probability for development of loss of response (LOT) to anti-tumor necrosis factor (TNF) agent stratified by treatment strategy. Combination therapy is presented with dotted line, monotherapy with solid line. study was conducted in a single center, a total of 116 patients with IBD were included with an average long-term follow-up duration of 159 weeks. The study confirmed that the concomitant IMM strategy to prevent LOR occurrence may not have a long-term favorable effect for IBD patients within various clinical settings.

In previous studies, the effects of combination therapy with IMMs for the prevention of the development of LOR to anti-TNF agents have yielded conflicting results for CD patients. A study conducted in Netherlands found that when using methotrexate as a concomitant medication, a higher response rate to infliximab was seen at the 1-year follow-up in CD patients [10]. On the other hand, combination therapy with an anti-TNF agent and IMM showed no significant difference in the response rate for CD patients in a long-term study conducted in Canada [11]. In addition, a recent meta-analysis revealed that concomitant therapy with an IMM was not significantly different from monotherapy treatment in predicting the incidence of LOR to anti-TNF agents in CD patients [12]. On the contrary, in the SONIC trial, concomitant azathioprine was effective for the prevention of LOR to infliximab in patients with moderate to severe CD [4]. However, the population in the SONIC trial consisted of all IMM naïve patients who had been diagnosed within the previous 2 years [4]. Thus, the study was limited, and it was difficult to generalize with regards to patients for

Probability of LOR to anti-TNF: immunomodulator exposure group
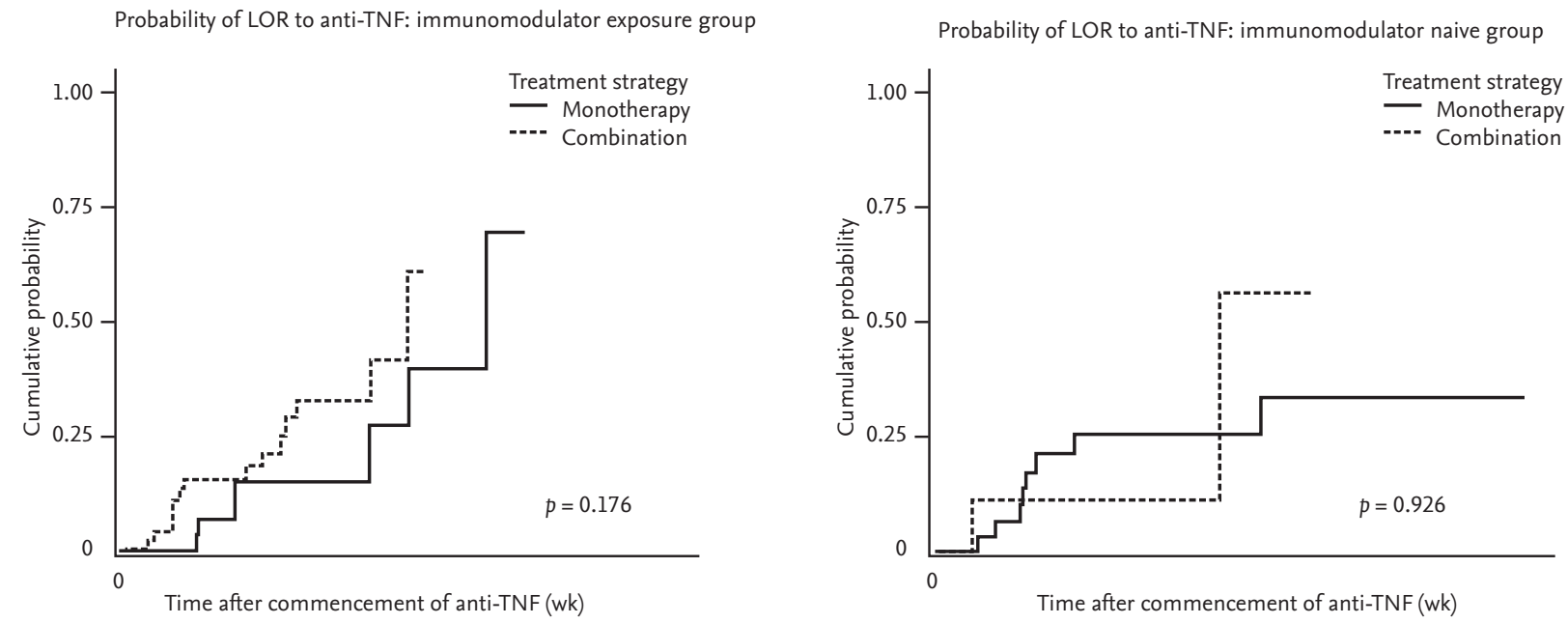

Figure 2. Kaplan-Meier curves show the probability for development of loss of response (LOT) to anti-tumor necrosis factor (TNF) agent for immunomodulator expose group (A), and naïve group (B). Combination therapy is presented with dotted line, monotherapy with solid line. 
Table 3. Univariate and multivariable analysis of factors associated with loss of response to anti-TNF

\begin{tabular}{|c|c|c|c|c|c|c|}
\hline \multirow{2}{*}{ Variable } & \multicolumn{3}{|c|}{ Univariate } & \multicolumn{3}{|c|}{ Multivariable } \\
\hline & HR & $95 \% \mathrm{CI}$ & $p$ value $^{a}$ & HR & $95 \% \mathrm{CI}$ & $p$ value \\
\hline Age & 1.011 & $0.985-1.037$ & 0.417 & & & \\
\hline \multicolumn{7}{|l|}{ Sex } \\
\hline Male & 1 & & & 1 & & \\
\hline Female & 3.777 & $1.1667-8.560$ & 0.001 & 3.032 & $1.467-6.268$ & $0.003^{\mathrm{a}}$ \\
\hline \multicolumn{7}{|l|}{ IBD, type } \\
\hline Crohn's disease & 1 & & & 1 & & \\
\hline Ulcerative colitis & 1.665 & $0.703-3.942$ & 0.247 & 2.022 & $0.984-4.157$ & 0.055 \\
\hline \multicolumn{7}{|l|}{ Combination therapy } \\
\hline Anti-TNF only & 1 & & & 1 & & \\
\hline Anti-TNF + IMM & 2.173 & $0.924-5.107$ & 0.075 & 1.972 & $0.927-4.193$ & 0.078 \\
\hline \multicolumn{7}{|l|}{ Anti-TNF, class } \\
\hline Infliximab & 1 & & & & & \\
\hline Adalimumab & 0.892 & $0.263-3.028$ & 0.892 & & & \\
\hline Golimumab & 5.254 & $1.024-26.946$ & 0.047 & & & \\
\hline \multicolumn{7}{|l|}{ IMM, exposure } \\
\hline No & 1 & & & & & \\
\hline Yes & 0.619 & $0.249-1.540$ & 0.302 & & & \\
\hline \multicolumn{7}{|l|}{ Abdominal surgery } \\
\hline No & 1 & & & & & \\
\hline Yes & 1.551 & $0.325-7.404$ & 0.582 & & & \\
\hline
\end{tabular}

TNF, tumor necrosis factor; HR, hazard ratio; CI, confidence interval; IBD, inflammatory bowel disease; IMM, immunomodulator.

${ }^{a} \mathrm{p}<0.05$.

whom thiopurine treatment failed or had the disease for a longer period of time. Further long-term clinical studies in $\mathrm{CD}$ patients are required to demonstrate the efficacy of combination therapy within various clinical settings.

The benefit of combination therapy with anti-TNF agents and IMMs was also equivocal in patients with UC. In a study conducted by Dumitrescu et al. [13], the concomitant use of IMMs at the point of starting infliximab was not associated with a sustained clinical response, but the introduction of an IMM at the time of dose doubling intensification was significantly associated with a lower risk of relapse for UC patients. Hence, additional clinical studies are also required to prove the efficacy of combination therapy for UC patients in various clinical situations, such as failed thiopurine treatment, longer durations of disease, or differing timings of IMM introduction.

Only female sex was significantly associated with the development of LOR to anti-TNF agents in the multivariable analysis. In this study, the significant difference in LOR incidence between males and females may be due to differences in adherent rates to anti-TNF agents. To confirm whether this was a biased result, the baseline characteristics stratified by sex are presented in Supplementary Table 1 . The adverse events related to anti-TNF agents were more common in females and the treatment period for the anti-TNF agents in males was longer than that of females, although this was not significant. Considering these findings, there may have been difference in adherence rates to anti-TNF agents between males and females in this study. A previous study demonstrated that non-adherence to ant-TNF agents is significantly associated with the incidence of LOR to an- 
ti-TNF agents [14]. A study conducted in the Netherlands revealed that drug persistence for anti-TNF in females was lower than that of males due to a higher rate of side effects in females [15]. The findings of these studies can serve as bases to support our hypothesis. Our hypothesis is limited because this study did not assess the adherence rate of each patient, but this is a plausible explanation for the higher incidence of LOR in females.

There were several limitations in this study. First, this was a retrospective study, and thus has inherent limitations. The baseline characteristic of patients included in the monotherapy or combination group were significantly different in terms of age and follow-up duration. We could not control for these differences, and they could be confounding factors and thus affect the results. According to previous studies, exposure to IMMs was a significant factor associated with the response rate of anti-TNF agents. However, there was a significant difference in the proportion of patients exposed to IMMs between the monotherapy and the combination groups. Finally, in the management of patients with LOR and the assessment of drug levels and antibodies is important to distinguish immunologic causes from non-immunologic causes. However, we could not confirm the presence of antibodies to anti-TNF agents or perform therapeutic drug monitoring. Hence, patients with LOR as a result of non-immunological causes could be included in this study and may act to underestimate the effect of the combination therapy.

In conclusion, the combination of anti-TNF agents and IMMs did not prove efficacious in the prevention of the development of LOR in IBD patients in this study. Only female sex was significantly associated with the development of LOR in IBD patients. Further long-term prospective studies are required to confirm these results.

\section{KEY MESSAGE}

1. Combination therapy with anti-tumor necrosis factor (TNF) agents and immunomodulators is not superior to anti-TNF treatment alone for preventing the development of loss of response (LOR) in inflammatory bowel disease (IBD) patients.

2. Only female sex was significantly associated with the development of LOR in IBD patients.

\section{Conflict of interest}

No potential conflict of interest relevant to this article was reported.

\section{REFERENCES}

1. Neurath MF. Current and emerging therapeutic targets for IBD. Nat Rev Gastroenterol Hepatol 2017;14:269-278.

2. Roda G, Jharap B, Neeraj N, Colombel JF. Loss of response to anti-TNFs: definition, epidemiology, and management. Clin Transl Gastroenterol 2016;7:e135.

3. Ben-Horin S, Chowers Y. Review article: loss of response to anti-TNF treatments in Crohn's disease. Aliment Pharmacol Ther 2011;33:987-995.

4. Colombel JF, Sandborn WJ, Reinisch W, et al. Infliximab, azathioprine, or combination therapy for Crohn's disease. N Engl J Med 2010;362:1383-1395.

5. Panaccione R, Ghosh S, Middleton S, et al. Combination therapy with infliximab and azathioprine is superior to monotherapy with either agent in ulcerative colitis. Gastroenterology 2014;146:392-400.

6. Jones JL, Kaplan GG, Peyrin-Biroulet L, et al. Effects of concomitant immunomodulator therapy on efficacy and safety of anti-tumor necrosis factor therapy for Crohn's disease: a meta-analysis of placebo-controlled trials. Clin Gastroenterol Hepatol 2015;13:2233-2240.

7. Billiet T, Cleynen I, Ballet V, et al. Prognostic factors for long-term infliximab treatment in Crohn's disease patients: a 20-year single centre experience. Aliment Pharmacol Ther 2016;44:673-683.

8. Varma P, Rajadurai AS, Holt DQ, et al. Immunomodulator use does not prevent first loss of response to anti-tumour necrosis factor alpha therapy in inflammatory bowel disease: long-term outcomes in a real-world cohort. Intern Med J 2019;49:753-760.

9. Satsangi J, Silverberg MS, Vermeire S, Colombel JF. The Montreal classification of inflammatory bowel disease: controversies, consensus, and implications. Gut 2006;55:749-753.

10. Hommes DW, van de Heisteeg BH, van der Spek M, Bartelsman JF, van Deventer SJ. Infliximab treatment for Crohn's disease: one-year experience in a Dutch academic hospital. Inflamm Bowel Dis 2002;8:81-86.

11. Teshima CW, Thompson A, Dhanoa L, Dieleman LA, Fedorak RN. Long-term response rates to infliximab 
therapy for Crohn's disease in an outpatient cohort. Can J Gastroenterol 2009;23:348-352.

12. Qiu Y, Chen BL, Mao R, et al. Systematic review with meta-analysis: loss of response and requirement of anti-TN$\mathrm{F} \alpha$ dose intensification in Crohn's disease. J Gastroenterol 2017;52:535-554.

13. Dumitrescu G, Amiot A, Seksik P, et al. The outcome of infliximab dose doubling in 157 patients with ulcerative colitis after loss of response to infliximab. Aliment Pharmacol Ther 2015;42:1192-1199.
14. van der Have M, Oldenburg B, Kaptein AA, et al. Non-adherence to anti-TNF therapy is associated with illness perceptions and clinical outcomes in outpatients with inflammatory bowel disease: results from a prospective multicentre study. J Crohns Colitis 2016;10:549-555.

15. Schultheiss JPD, Brand EC, Lamers E, et al. Earlier discontinuation of TNF- $\alpha$ inhibitor therapy in female patients with inflammatory bowel disease is related to a greater risk of side effects. Aliment Pharmacol Ther 2019;50:386-396. 
Supplementary Table 1. Characteristic of included patients stratified by sex

\begin{tabular}{|c|c|c|c|}
\hline Characteristic & Male $(\mathrm{n}=75)$ & Female $(n=41)$ & $p$ value \\
\hline Age, yr & $34.37 \pm 15.08$ & $33.44 \pm 14.67$ & 0.748 \\
\hline Body weight at time of initiation, kg & $60.6 \pm 13.1$ & $49 \cdot 5 \pm 8.2$ & $0.000^{\mathrm{a}}$ \\
\hline IBD type & & & 0.607 \\
\hline Crohn's disease & $50(66.6)$ & $30(73.17)$ & \\
\hline Ulcerative colitis & $25(33 \cdot 3)$ & $11(26.8)$ & \\
\hline Anti-TNF treatment duration & $170.57 \pm 92.73$ & $137.80 \pm 80.49$ & 0.059 \\
\hline Anti-TNF type & & & 0.171 \\
\hline Infliximab & $58(77 \cdot 3)$ & $37(90.2)$ & \\
\hline Adalimumab & $14(18.7)$ & $4(9.8)$ & \\
\hline Golimumab & $3(4 \cdot 0)$ & o & \\
\hline Combination therapy & $39(52.0)$ & $16(39.0)$ & 0.253 \\
\hline Immunomodulator type & & & 0.057 \\
\hline Azathioprine & $32(82.1)$ & $10(62.5)$ & \\
\hline 6-Mercaptopurine & $7(17.9)$ & $4(25 \cdot 0)$ & \\
\hline Methotrexate & 0 & $2(12.5)$ & \\
\hline History of abdominal surgery & $7(9 \cdot 3)$ & $2(4 \cdot 9)$ & 0.621 \\
\hline Steroid exposure & $69(98.6)$ & $41(100)$ & 1.000 \\
\hline Anti-TNF discontinuation & $15(20.0)$ & $20(48.8)$ & $0.004^{\mathrm{a}}$ \\
\hline Loss of response & $14(18.7)$ & $17(41.5)$ & \\
\hline Adverse events & $1(1.3)$ & $3(7 \cdot 3)$ & \\
\hline
\end{tabular}

Values are presented as mean $\pm \mathrm{SD}$ or number (\%).

IBD, inflammatory bowel disease; TNF, tumor necrosis factor.

${ }^{\mathrm{a}} \mathrm{p}<0.05$. 